\title{
Hernia de Amyand. Reporte de un caso y revisión de la literatura
}

\author{
Amyand's Hernia. Case report and literature review
}

Gómez-Sánchez Javier, García-Rubio Jesús, Garde-Lecumberri Carlos, Mirón-Pozo Benito

Hospital Campus de la Salud, Granada, España.

\begin{abstract}
Resumen
Las hernias inguinales constituyen una de las entidades más frecuentes en cirugía. La localización de una apendicitis en el seno de una hernia inguinal, se conoce como hernia de Amyand, una patología poco frecuente, en torno al $0,13 \%$, que requiere de un tratamiento quirúrgico urgente. A continuación se presenta el caso de un paciente con una hernia de Amyand, su clínica y diagnóstico con el objetivo de exponer su actitud terapéutica.
\end{abstract}

\section{Abstract}

Inguinal hernias are one of the most frequent pathologies in surgery. The location of an appendicitis in an inguinal hernia is known as Amyand's hernia, a rare condition, around $0.13 \%$, which requires urgent surgical treatment. We present the case of a patient with a hernia of Amyand, the clinic and diagnosis with the aim of exposing his therapeutic attitude.
Palabras clave: Hernia, inguinal, Amyand.

Keywords: Hernia, inguinal, Amyand.

\section{INTRODUCCIÓN}

La presencia de la patología herniaria inguinal es una de las más frecuentes en nuestro medio, estimándose una prevalencia del $15 \%$ en adultos, con una proporción de hombres respecto a mujeres de $12 / 1$. El contenido de estas hernias suele ser con frecuencia de grasa epiploica intraabdominal, asas de intestino delgado, colon y en menor proporción vejiga (1). Es infrecuente hallar el apéndice vermiforme en el saco herniario, y con menos probabilidad, que este se encuentre inflamado en su interior. La aparición de esto se conoce como hernia de Amyand, y supone la combinación de dos de las patologías más habituales en cirugía: la hernia inguinal y la apendicitis aguda.

Su debut clínico suele ser una tumoración inguinal dolorosa compatible con una hernia inguinal incarcerada, as como una historia clínica de fiebre y malestar general como pródromo, si bien esto no es constante.

Su diagnóstico prequirurgico es infrecuente, pero si se detecta, nos permite planificar con antelación el tratamiento que se va a efectuar (2).

\section{CASO CLÍNICO}

Se presenta el caso de un varón de 56 años que acude al servicio de urgencias por clínica de un día de evolución de febrícula de hasta 37,8 grados, astenia, y tumoración inguinal dolorosa con signos floglóticos a nivel de la piel. No presenta alteraciones miccionales ni alteraciones del tránsito intestinal, negando vómitos, estreñimiento o diarrea. Tampoco asocia otra clínica por aparatos o sistemas.

Analíticamente destacaba una leucocitosis $\left(17,30 \times 10^{3 /}\right.$ $\mu \mathrm{L}$ ) con neutrofilia ( $83 \%$ neutrófilos) con una elevación de la proteína $\mathrm{C}$ reactiva.

A la exploración presentaba un abdomen blando y depresible, con molestias a la palpación en hipogastrio y fosa iliaca derecha, asociando cierta irritación peritoneal. Así mismo, encontramos una tumoración inguinal derecha irreductible, dolorosa a la palpación.

Ante la discordancia de la historia clínica con la exploración, completamos el estudio mediante la realización de una ecografía, hallándose una hernia inguinal derecha que contenía una estructura tubular de $9 \mathrm{~mm}$ con su pared engrosada, compatible con apéndice vermiforme, que muestra hiperemia, cambios inflamatorios de la grasa adyacente y una imagen hiperecogénica con sombra acústica posterior en su interior, compatible con apendicolito, todo ello compatible con apendicitis aguda.

Se completó el diagnóstico mediante un TAC con contraste intravenoso que mostró el apéndice vermiforme engrosado, con origen en el ciego extendiéndose hacia el canal inguinal. (Figura 1 y 2 - ver en la página siguiente) 


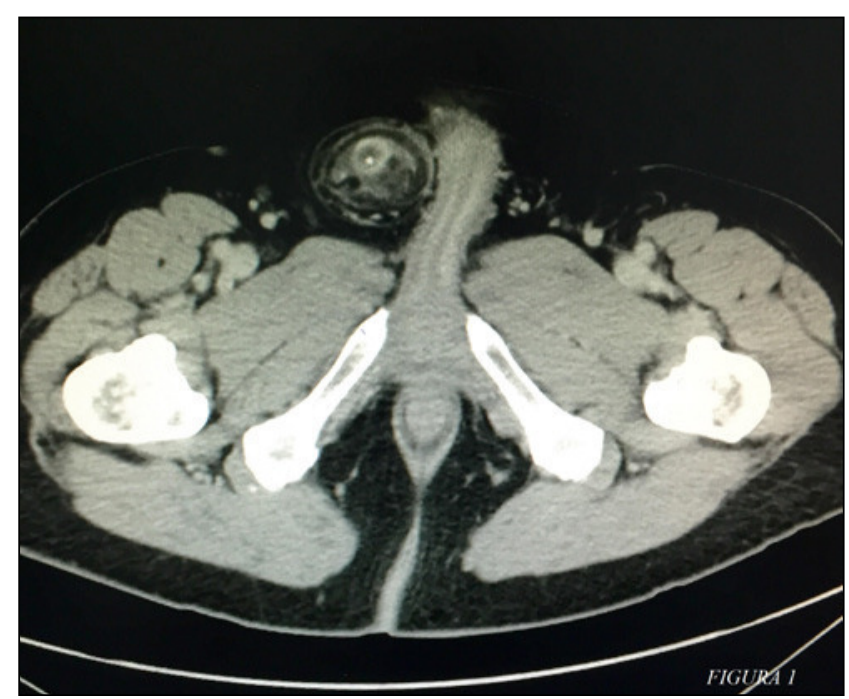

Figura 1.

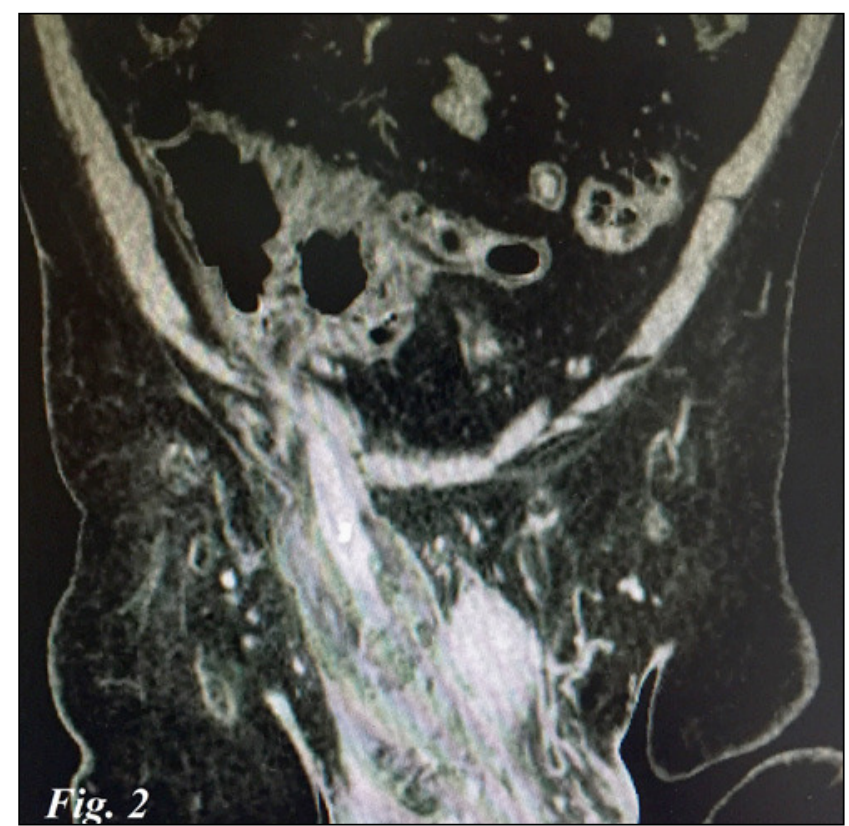

Figura 2.

Se decide intervenir quirúrgicamente, accediendo mediante vía inguinal anterior, donde se encuentra una tumoración dura, irreductible, compatible con hernia inguinal indirecta tipo 3 de Gilbert. Se diseca su saco herniario, y se procede a la apertura del mismo (previo aislamiento del campo con compresas betadinadas), visualizando en él un apéndice de aspecto gangrenoso (figura 3), sin líquido libre purulento a su alrededor, así como engrosamiento del mesoapéndice adherido al saco herniario.

Se realiza apendicectomía por el abordaje inguinal, con posterior resección y cierre del saco herniario, que se reduce a la cavidad abdominal. Tras esto, se procede a realizar hernioplastia inguinal según técnica de Lichtenstein con malla de polipropileno.

El postoperatorio del paciente transcurre sin incidencias, siendo dado de alta a los dos días tras la cirugía. El informe anatomopatólogico confirma la presencia del apéndice vermiforme gangrenoso, parcialmente desestructurado, sin signos de malignidad.

En la última revisión, el paciente no ha presentado recidiva herniaria, ni presenta signos de infección en la prótesis de polipropileno.

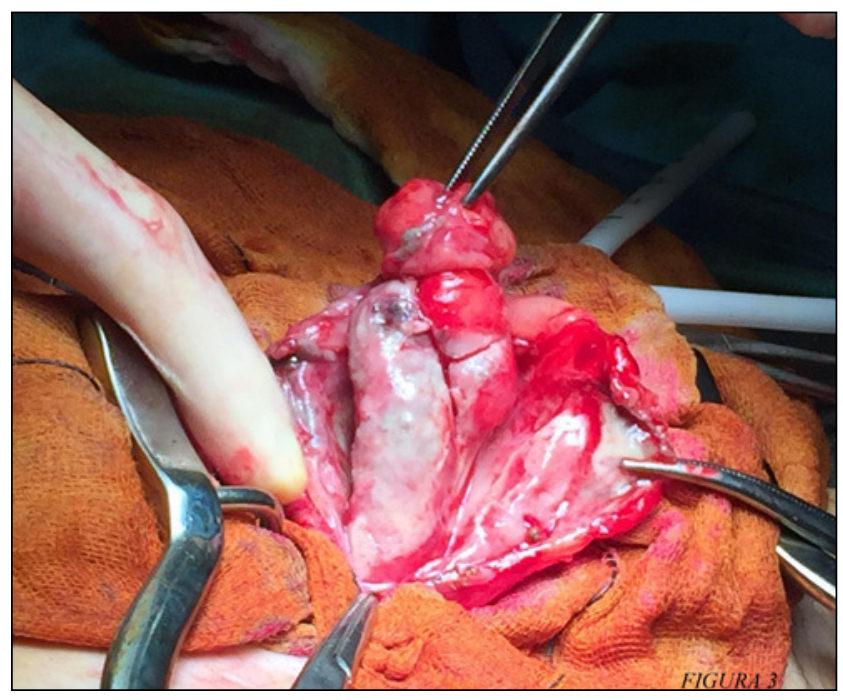

Figura 3.

\section{DISCUSIÓN}

Las hernias inguinales constituyen una de las patologías más frecuentes en la práctica quirúrgica, siendo estas clasificadas por sus consideraciones anatómicas y funcionales. La clasificación más usada es la de Gilbert modificada (3), donde se sistematizan las hernias como:

- Tipo 1. Hernia indirecta, con anillo menor de $2 \mathrm{~cm}$.

- Tipo 2. Hernia indirecta con anillo entre 2 y $4 \mathrm{~cm}$.

- Tipo 3. Hernia indirecta con anillo dilatado mayor de $4 \mathrm{~cm}$.

- Tipo 4. Hernia directa, con fallo completo de toda la pared del canal inguinal.

- Tipo 5. Hernia directa, con fallo diverticular de la pared.

- Tipo 6. Hernia mixta (directa e indirecta, también conocida como "en pantalón").

- Tipo 7. Hernia crural.

Esta clasificación sin embargo no se atiene al contenido que compone el saco herniario, por lo que cuando nos encontramos ante una hernia de Amyand, podría ser útil el uso de la clasificación de Losanoff y Basson (4), que se basa en el grado de inflamación del apéndice dentro del saco herniario:

- Tipo 1. Con apéndice normal.

- Tipo 2. Con apendicitis aguda localizada dentro del saco.

- Tipo 3. Con apendicitis aguda y peritonitis

- Tipo 4. Con apendicitis y otro hallazgo intraabdominal (como sería un mucocele apendicular, pseudomixoma apendicular...)

La hernia de Amyand constituye la suma de dos de las patologías más frecuentes en cirugía, como son la hernia inguinal y la apendicitis aguda, de forma simultánea, algo poco común pero que debe tenerse en cuenta a la hora de establecer una actitud terapéutica. Su incidencia se estima alrededor del $0,1 \%$ aunque si bien esta cifra no discrimina entre en las hernias que presentan el apéndice sin signos de inflamación de las que contienen una apendicitis aguda. 
Fue descrita por primera vez en 1735 por Claudius Amyand, en un varón de 11 años que presentaba una fístula inguinal asociada a una hernia, hallando en la cirugía que esto era causado por el apéndice perforado.

Para su diagnóstico, además de la anamnesis y exploración, es necesario un hemograma completo donde aparece leucocitosis con neutrofilia, y suele ser útil la realización de una prueba de imagen (ecografía o bien TAC) que confirme la presencia del apéndice con signos de inflamación en el saco herniario, además de ayudar a esclarecer el abordaje quirúrgico (5).

A día de hoy no existe consenso sobre cuál es su abordaje ideal, bien por un acceso inguinal anterior, o bien por un acceso laparotómico, pero en cualquiera de ambos se debe de tener la suficiente comodidad para realizar la apendicetomía además de reparar el defecto herniario.

Un punto en el que parecen coincidir las publicaciones $(6,7)$ es en la resolución del defecto herniario en el mismo acto quirúrgico, sin quedar tampoco claro el método de reparación, postulándose diversas técnicas, bien sin uso de material protésico por el riesgo de infección del mismo (secundaria a la proliferación bacteriana de la apendicitis) o bien mediante la colocación de una malla de polipropileno que permita reparar la hernia.

En nuestro caso se optó por la colocación de una malla de polipropileno que corrigiese el defecto de la pared posterior del canal inguinal, dado que la inflamación e infección se encontraba delimitada al saco herniario y al apéndice, mientras que el canal inguinal no presentaba signos de infección o peritonitis.

El porcentaje de infección de la herida quirúrgica se sitúa en torno al 5,5\% aunque haya series que presenten hasta el $50 \%$ (7). La mortalidad de este tipo de hernias viene derivada de la posible perforación apendicular o demora en su diagnóstico que desemboque en una difusión peritoneal de la infección, situada en torno al $15 \%$ y el $30 \%$. (7)

\section{CONCLUSIONES}

La hernia de Amyand se trata de una patología poco frecuente, cuyo diagnóstico rara vez es previo a la cirugía dada su clínica inespecífica, pero que debemos tener en cuenta ante la presencia de una hernia inguinal complicada, por el riesgo que puede suponer para la integridad del paciente el hecho de no realizar la apendicectomía, y por la ausencia de consenso sobre que técnica es la idónea para la reparación herniaria, dado el riesgo de infección protésica y recidiva.

\section{REFERENCIAS BIBLIOGRÁFICAS}

1. Moreales S, Barreiro F, Hernandez P, Feliu X. Cirugía de la Pared Abdominal. 2o Edición. Arán Ediciones, Madrid, 2013.

2. Karatas A, Makay O, Salihoglu Z. Can preoperative diagnosis affect the choice of treatment in Amyand's hernia? Report of a case. Hernia. 2009; 13(2):.225-27.

3. Romero Vargas ME, Palacios García E, del Álamo Juzgado C Herrera Gutiérrez L, Roldán Aviña JP, Muñoz Pozo F. Clasificación anatómica de las hernias de pared abdominal. Cir. Andal. 2013; 24: 222-24

4. Losanoff JE, Basson MD. Amyand hernia: A classification to improve management. Hernia. 2008; 12(3): 325-26.

5. Agirre Etxabe L, Prieto Calvo M, García Etxebarría A, García González JM, Sarriugarte Lasarte A. Colina Alonso A. Hernia de Amyand (tipo 2 de Losanoff) diagnosticada preoperatoriamente y tratada mediante hernioplastia con malla biológica. Rev Hispanoamer Hernia. 2014; 2(4):169-72.
6. Kueper M.A, Kirschniak A, Ladurner R, Granderath FA, Konigsrainer $A$. Incarcerated recurrent inguinal hernia with covered and perforated appendicitis and periappendicular abscess: Case report and review of the literature. Hernia. 2007; 11(2): 189-91.

7. Sharma H, Gupta A, Shekhawat NS, Memon B, Memon MA. Amyand's hernia: A report of 18 consecutive patiens over 15 year period. Hernia. 2007; 11(1): 31-35. 